\title{
THE DIVERGENCE THEOREM ${ }^{1}$
}

\author{
W. F. PFEFFER
}

\begin{abstract}
We define a well-behaved multidimensional Riemann type integral such that the divergence of any vector field continuous in a compact interval and differentiable in its interior is integrable, and the integral equals the flux of the vector field out of the interval.
\end{abstract}

1. Introduction. The existence of a derivative which is not Lebesgue integrable was probably the main driving force behind the work of Denjoy (1912) and Perron (1914) who, independently and by different means, defined an integral which integrates the derivative of any differentiable function. A corresponding local result in higher dimensions should be the integrability of the divergence of any differentiable vector field. It is remarkable that among the many higher dimensional generalizations of the Denjoy-Perron integral produced during the last 70 years, none lives up to this requirement; we quote $\left[\mathbf{K}_{\mathbf{2}}\right.$ and $\left.\mathbf{M c}\right]$ as the most recent examples. Notable exceptions are the papers $\left[\mathbf{M}_{1}\right.$ and $\mathbf{M}_{2}$ ] of J. Mawhin, which came to the author's attention only after the major bulk of this work had been completed. However, Mawhin's integrals lack some standard properties one normally expects of any integral; e.g., the integrability over each member of a finite division of an interval does not imply integrability over the whole interval (for more details see 7.2 and 7.3 , and also the "Added in the proof" section).

Elaborating on the ideas of Henstock and Kurzweil (cf. [ $\mathbf{H}_{3}$ and $\left.\mathbf{K}_{1}\right]$ ), we define a Riemann-type integral over $m$-dimensional compact intervals which has all the usual properties of integrals, and for which a very general divergence theorem holds (Theorem 5.4). For nonnegative functions the integral coincides with the Lebesgue integral, and for $m=1$ it is equal to the Henstock-Kurzweil integral of $\left[\mathbf{H}_{\mathbf{3}}\right]$ and hence also to the classical Denjoy-Perron integral (see $[\mathbf{L W}]$ ).

Our results are achieved by employing partitions of a Vitali type. Since the regularity of an interval is by no means a hereditary property, the Vitali condition cannot be applied directly (as in $\left[\mathbf{M}_{1}\right.$ and $\left.\mathbf{M}_{\mathbf{2}}\right]$ ). Rather, we have to consider a relative Vitali condition with respect to a finite family of planes parallel to the coordinate axes. Roughly speaking, an interval $A$ is regular relative to a plane $H$ intersecting $A$ if $A \cap H$ is absolutely regular in $H$ and the diameters of $A$ and $A \cap H$ are equal. In the figure below neither $A$ nor $B$ is absolutely regular, but $A$ is regular relative to $H$ while $B$ is not.

As the use of intervals precludes all but trivial formulations of the Stokes theorem (see 6.2), global results are not accessible in the present setting. On the other hand,

Received by the editors April 9, 1985.

1980 Mathematics Subject Classification. Primary 26B20, 26B15; Secondary 26A39.

${ }_{1}^{1}$ This work was done at the University of Petroleum and Minerals (UPM) in Dhahran, Saudi Arabia, and was partially supported by UPM Research Project MATH/MANIFOLD/68. The final version of the paper was written at the University of the Witwatersrand in Johannesburg, South Africa. 


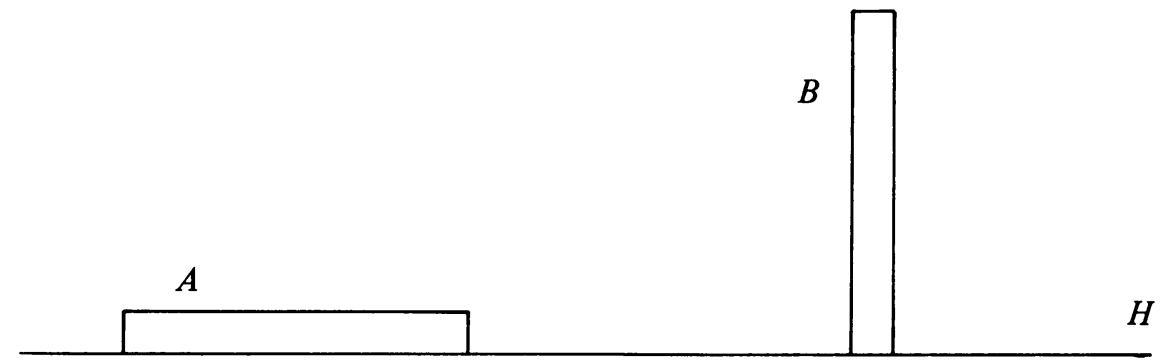

it seems that a reasonably general Stokes theorem for piecewise linear manifolds can be obtained by our method if convex linear cells are used instead of intervals. Such a change is nontrivial and leads to problems of a different nature than those discussed in this paper (cf. 6.3). Whether a satisfactory Stokes theorem can be established for differentiable manifolds was an open problem when this paper was written. Since then two different solutions have been obtained independently in [JK and $\mathbf{P}_{\mathbf{2}}$.

The present paper is organized as follows. After some preliminary definitions and lemmas in $\S 2$, the integral is defined in $\S 3$, where its basic properties are proved. $\S 4$ deals with the relationship to the Lebesgue integral and resulting convergence theorems. The divergence theorem is proved in $\S 5$. In $\S 6$ a modest proposition about the change of variable is established. $\S 7$ is devoted to an informal discussion of some alternative definitions of the integral.

2. Preliminaries. By $\mathbf{R}$ and $\mathbf{R}_{+}$we denote the sets of all real and all positive real numbers, respectively. All functions in this paper are real-valued, and the constant functions are tacitly identified with the elements of $\mathbf{R}$. Often, a function on a set $A$ and its restriction to a set $B \subset A$ are denoted by the same symbol. The algebraic operations, partial order, and convergence among functions on the same set are defined pointwise.

Throughout this paper, $m \geq 1$ is a fixed integer, and $\mathbf{R}^{m}$ denotes the $m$ dimensional Euclidean space. If $x=\left(\xi_{1}, \ldots, \xi_{m}\right)$ is an element of $\mathbf{R}^{m}$, we let $|x|=$ $\max \left\{\left|\xi_{1}\right|, \ldots,\left|\xi_{m}\right|\right\}$. In $\mathbf{R}^{m}$ we use exclusively the metric induced by the norm $|x|$. The distance between a point $x \in \mathbf{R}^{m}$ and a set $E \subset \mathbf{R}^{m}$ is denoted by $\operatorname{dist}(x, E)$. If $E \subset \mathbf{R}^{m}$, then $E^{\circ}, \partial E, d(E)$, and $|E|$ denote, respectively, the interior, boundary, diameter, and outer measure of $E$. We note that when no further specification is given, the words "outer measure", "measure", and "measurable", as well as the expression "almost all", always refer to the $m$-dimensional Lebesgue measure in $\mathbf{R}^{m}$.

Let $k$ be an integer with $0 \leq k \leq m-1$. By a plane, or more precisely a $k$-plane, we mean a $k$-dimensional linear submanifold of $\mathbf{R}^{m}$ which is parallel to $k$ distinct coordinate axes. If $E$ is a subset of a $k$-plane and $k \geq 1$, we denote by $|E|_{k}$ the $k$-dimensional outer Lebesgue measure of $E$. If $E \subset \mathbf{R}^{m}$ is finite, then $|E|_{0}$ denotes the number of elements of $E$.

An interval is a nondegenerate compact rectangle in $\mathbf{R}^{m}$, i.e., the set $A=$ $\prod_{i=1}^{m}\left[a_{i}, b_{i}\right]$, where $a_{i}, b_{i} \in \mathbf{R}$ and $a_{i}<b_{i}, i=1, \ldots, m$. If $b_{1}-a_{1}=\cdots=b_{m}-a_{m}$, then the interval $A$ is called a cube. 
Let $A$ be an interval and let $\sharp$ be a family of planes. The absolute regularity of $A$ is the number $r(A)$ defined by $r(A)=|A| /[d(A)]^{m}$ (cf. [S, Chapter $4, \S 2$, p. 106]). Clearly, $0<r(A) \leq 1$, and $r(A)=1$ if and only if $A$ is a cube. The regularity of $A$ relative to $\mathcal{H}$ is the number $r(A ; \mathcal{H})$ defined as follows: if $\mathcal{H}=\varnothing$, then $r(A ; \mathscr{H})=r(A)$; if $\mathscr{H}$ consists of a single $k$-plane $H$, then $r(A ; \mathscr{H})=|A \cap H|_{k} /[d(A)]^{k}$ whenever $A \cap H \neq \varnothing$, and $r(A ; \not{H})=r(A)$ otherwise; finally, if $\mathscr{H} \neq \varnothing$ is arbitrary, then

$$
r(A ; \not{H})=\sup \{r(A ;\{H\}): H \in \not{H}\} .
$$

2.1. For any interval $A$ the following conditions hold:

(i) If $\not{H}$ is a family of planes, then $r(A) \leq r(A ; \not{H}) \leq 1$. In particular, $r\left(A ; \nvdash^{*}\right) \leq$ $r(A ; \mathscr{H})$ for each family $\mathscr{H}^{*} \subset \mathcal{H}$.

(ii) If $H$ and $H^{*}$ are planes, $H^{*} \subset H$, and $A \cap H^{*} \neq \varnothing$, then $r(A,\{H\}) \leq$ $r\left(A,\left\{H^{*}\right\}\right)$. In particular, $r\left(A ;\left\{H^{*}\right\}\right)=1$ whenever $H^{*}$ is a 0 -plane.

Indeed, if $k$ and $k^{*}$ are, respectively, the dimensions of $H$ and $H^{*}$, then

$$
\begin{aligned}
r(A) & =\frac{|A|}{[d(A)]^{m}} \leq \frac{|A \cap H|_{k}[d(A)]^{m-k}}{[d(A)]^{m}}=r(A ;\{H\}) \\
& \leq \frac{\left|A \cap H^{\prime}\right|_{k^{*}}[d(A)]^{k-k^{*}}}{[d(A)]^{k}}=r\left(A ;\left\{H^{*}\right\}\right) .
\end{aligned}
$$

We say that intervals $A$ and $B$ overlap whenever $A^{\circ} \cap B^{\circ} \neq \varnothing$. A division of an interval $A$ is a finite family of nonoverlapping intervals whose union is $A$. $\mathrm{A}$ partition of an interval $A$ is a set $\left\{\left(A_{1}, x_{1}\right), \ldots,\left(A_{p}, x_{p}\right)\right\}$ such that $\left\{A_{1}, \ldots, A_{p}\right\}$ is a division of $A$, and $x_{i} \in A_{i}, i=1, \ldots, p$.

2.2. Definition. Let $P=\left\{\left(A_{1}, x_{1}\right), \ldots,\left(A_{p}, x_{p}\right)\right\}$ be a partition of an interval $A$, let $\varepsilon$ and $\delta$ be functions on $A$, and let $\mathcal{H}$ be a family of planes.

(i) If $d\left(A_{i}\right)<\delta\left(x_{i}\right), i=1, \ldots, p$, we call $P \delta$-fine.

(ii) If $r\left(A_{i} ; \not{H}\right) \geq \varepsilon\left(x_{i}\right), i=1, \ldots, p$, we call $P$ an $(\varepsilon, \not H)$-partition.

By the above definition we control the size and shape of the intervals of a partition. Part (i) is due to Henstock, Kurzweil, and McShane; part (ii), which plays an essential role when $m \geq 2$, is new (cf. [ $\mathbf{M}_{2}$, Definitions 5 and 9]). The family of all $\delta$-fine $(\varepsilon, \mathscr{H})$-partitions of an interval $A$ is denoted by $\mathcal{P}(A ; \varepsilon, \mathscr{H} ; \delta)$. In view of 2.1(i), we have $\mathcal{P}(A ; \varepsilon, \not{H} ; \delta) \subset \mathcal{P}\left(A ; \varepsilon^{*}, \mathfrak{H}^{*} ; \delta^{*}\right)$ whenever $\varepsilon^{*} \leq \varepsilon, \not{H} \subset \mathcal{H}^{*}$, and $\delta \leq \delta^{*}$.

We close this section by proving a pivotal proposition about the existence of a $\delta$-fine $(\varepsilon, \mathcal{H})$-partition.

2.3. LemMA. Let $A$ be an interval and let $\varepsilon<1$. Then there is a division $D$ of $A$ such that $r(D)>\varepsilon$ for each $D \in D$.

PROOF. If $A=\prod_{i=1}^{m}\left[a_{i}, b_{i}\right]$, let $c_{i}=b_{i}-a_{i}, i=1, \ldots, m$, and choose an $\alpha>0$ so that $[(1-\alpha) /(1+\alpha)]^{m}>\varepsilon$. Find integers $p_{i} \geq 1$ with $\left|\left(c_{1} / c_{i}\right)\left(p_{i} / p_{1}\right)-1\right| \leq \alpha$, and set

$$
A_{j_{1} \ldots j_{m}}=\prod_{i=1}^{m}\left[a_{i}+j_{i} \frac{c_{i}}{p_{i}}, a_{i}+\left(j_{i}+1\right) \frac{c_{i}}{p_{i}}\right]
$$


where $j_{i}=0, \ldots, p_{i}-1$ for $i=1, \ldots, m$. Clearly, the intervals $A_{j_{1} \ldots j_{m}}$ form a division of $A$, and if $c_{k} / p_{k}=\max \left\{c_{1} / p_{1}, \ldots, c_{m} / p_{m}\right\}$, then

$$
\begin{aligned}
r\left(A_{j_{1} \ldots j_{m}}\right) & =\left(\frac{p_{k}}{c_{k}}\right)^{m} \prod_{i=1}^{m}\left(\frac{c_{i}}{p_{i}}\right)=\left(\frac{c_{1}}{c_{k}} \frac{p_{k}}{p_{1}}\right)^{m} \prod_{i=1}^{m}\left(\frac{c_{i}}{c_{1}} \frac{p_{1}}{p_{i}}\right) \\
& \geq\left(\frac{1-\alpha}{1+\alpha}\right)^{m}>\varepsilon .
\end{aligned}
$$

2.4. Proposition. Let $A$ be an interval. Then $\mathrm{P}(A ; 1-\delta, \varnothing ; \delta) \neq \varnothing$ for each $\delta: A \rightarrow \mathbf{R}_{+}$.

Proof. Let $B$ be the family of all intervals $B \subset A$ with $P(B ; 1-\delta, \varnothing ; \delta)=\varnothing$, and assume that $A \in B$. Using 2.3, find a division $D_{1}$ of $A$ such that $d(D)<2^{-1}$ and $r(D)>1-2^{-1}$ for each $D \in D_{1}$. If for every $D \in D_{1}$ there is a $P_{D} \in \mathcal{P}(D ; 1-\delta, \varnothing ; \delta)$, then contrary to our assumption, $P=\bigcup_{D \in D_{1}} P_{D}$ belongs to $P(A ; 1-\delta, \varnothing ; \delta)$. Thus some $D_{1} \in D_{1}$ belongs to $B$. Using 2.3 again, find a division $D_{2}$ of $D_{1}$ such that $d(D)<2^{-2}$ and $r(D)>1-2^{-2}$ for each $D \in D_{2}$, and as before show that some $D_{2} \in D_{2}$ belongs to $B$. Proceeding inductively, we construct a decreasing sequence $\left\{D_{n}\right\}$ in $B$ with $d\left(D_{n}\right)<2^{-n}$ and $r\left(D_{n}\right)>1-2^{-n}, n=1,2, \ldots$. Now if $\left\{x_{0}\right\}=\bigcap_{n=1}^{\infty} D_{n}$ and $2^{-n_{0}}<\delta\left(x_{0}\right)$, then $\left\{\left(D_{n_{0}}, x_{0}\right)\right\}$ belongs to $P\left(D_{n_{0}} ; 1-\delta, \varnothing ; \delta\right)$, a contradiction.

2.5. Corollary. Let $\varepsilon$ and $\delta$ be functions on an interval $A$, and let $\sharp$ be $a$ family of planes. Then $\mathcal{P}(A ; \varepsilon, H ; \delta) \neq \varnothing$ whenever $\varepsilon<1$ and $\delta>0$.

Except for $\S 7$, we shall use $(\varepsilon, \nvdash)$-partitions only when $\varepsilon$ is a constant function with $0<\varepsilon \leq \frac{1}{2}$, and the family $\mathcal{H}$ is finite. It will be convenient to call a regulator each pair $(\varepsilon, \not{H})$ where $\varepsilon \in\left(0, \frac{1}{2}\right]$ and $\not{\not}$ is a finite family of planes.

3. Definition of the integral and its basic properties. If $f$ is a function on an interval $A$, then for each partition $P=\left\{\left(A_{1}, x_{1}\right), \ldots,\left(A_{p}, x_{p}\right)\right\}$ of $A$ we set

$$
\sigma(f, P)=\sum_{i=1}^{p} f\left(x_{i}\right)\left|A_{i}\right| .
$$

3.1. Definition. Let $f$ be a function on an interval $A$. We say that $f$ is integrable on $A$ if there is a real number $I$ with the following property: given a regulator $(\varepsilon, \mathcal{H})$, we can find a $\delta: A \rightarrow \mathbf{R}_{+}$such that $|\sigma(f, P)-I|<\varepsilon$ for each $P \in P(A ; \varepsilon, \not H ; \delta)$.

It follows from 2.5 that the number $I$ of the previous definition is determined uniquely by the integrable function $f$. It is called the integral of $f$ over $A$ denoted by $\int_{A} f$. The family of all integrable functions on $A$ is denoted by $R(A)$. It is clear that each function $f$ on $A$ which is integrable in the sense of [Mc, $\S 1.6]$ belongs to $R(A)$, and that $\int_{A} f$ is equal to the integral defined in [Mc, ibid.]. Moreover, the two integrals coincide when $m=1$. Some motivating remarks regarding Definition 3.1 are given in $\S 7$.

3.2. Proposition. If $A$ is an interval, then $R(A)$ is a linear space, and the map $f \mapsto \int_{A} f$ is a nonnegative linear functional on $R(A)$.

The proposition is a direct consequence of Definition 3.1. 
3.3. LEMMA. Let $f$ be a function on an interval $A$. Then $f \in R(A)$ if and only if given a regulator $(\varepsilon, \mathcal{H})$, there is a $\delta: A \rightarrow \mathbf{R}_{+}$such that $|\sigma(f, P)-\sigma(f, Q)|<\varepsilon$ for each $P, Q \in P(A ; \varepsilon, \not H ; \delta)$.

ProOF. As the converse is obvious, suppose that $f$ satisfies the condition of the lemma. For $n=1,2, \ldots$, we can find $\delta_{n}: A \rightarrow \mathbf{R}_{+}$such that $|\sigma(f, P)-\sigma(f, Q)|<$ $2^{-n}$ for every $P, Q \in P\left(A ; \frac{1}{2}, \varnothing ; \delta_{n}\right)$. Replacing each $\delta_{n}$ by $\min \left\{\delta_{1}, \ldots, \delta_{n}\right\}$, we may assume that $\delta_{1} \geq \delta_{2} \geq \cdots$. Using 2.5 to select a $P_{n} \in P\left(A ; \frac{1}{2}, \varnothing ; \delta_{n}\right)$, we obtain a Cauchy sequence $\left\{\sigma\left(f, P_{n}\right)\right\}$. Let $I=\lim \sigma\left(f, P_{n}\right)$, and choose a regulator $(\varepsilon, \not H)$. Then there is an integer $k$ with $2^{-k}<\varepsilon / 3$ and $\left|\sigma\left(f, P_{k}\right)-I\right|<\varepsilon / 3$, and there is a $\delta: A \rightarrow \mathbf{R}_{+}$such that $\delta \leq \delta_{k}$ and $|\sigma(f, P)-\sigma(f, Q)|<\varepsilon / 3$ for each $P, Q \in \mathcal{P}(A ; \varepsilon, \mathcal{H} ; \delta)$. Use 2.5 to select a $Q \in \mathcal{P}\left(A ; \frac{1}{2}, \varnothing ; \delta\right)$, and observe that this $Q$ belongs to $P(A ; \varepsilon, \mathcal{H} ; \delta) \cap P\left(A ; \frac{1}{2}, \varnothing ; \delta_{k}\right)$. Thus for each $P \in \mathcal{P}(A ; \varepsilon, \mathcal{H} ; \delta)$, we have

$$
\begin{aligned}
|\sigma(f, P)-I| & \leq|\sigma(f, P)-\sigma(f, Q)|+\left|\sigma(f, Q)-\sigma\left(f, P_{k}\right)\right|+\left|\sigma\left(f, P_{k}\right)-I\right| \\
& <\varepsilon / 3+2^{-k}+\varepsilon / 3<\varepsilon,
\end{aligned}
$$

and so $f$ is integrable.

3.4. PROPOSITION. Let $f$ be a function integrable on an interval $A$. Then $f$ is integrable on each subinterval of $A$.

ProOF. Let $B$ be a subinterval of $A$, and let $(\varepsilon, \mathscr{H})$ be a regulator. By 3.3 , there is a $\delta: A \rightarrow \mathbf{R}_{+}$such that $\left|\sigma\left(f, P^{*}\right)-\sigma\left(f, Q^{*}\right)\right|<\varepsilon$ for each $P^{*}, Q^{*} \in \mathcal{P}(A ; \varepsilon, \mathscr{H} ; \delta)$. Choose intervals $B_{1}, \ldots, B_{n}$ so that $\left\{B, B_{1}, \ldots, B_{n}\right\}$ is a division of $A$, and use 2.5 to find $S_{i} \in P\left(B_{i} ; \varepsilon, \not H ; \delta\right), i=1, \ldots, n$. Now if $P, Q \in \mathcal{P}(B ; \varepsilon, \not H ; \delta)$, then $P^{*}=P \cup \bigcup_{i=1}^{n} S_{i}$ and $Q^{*}=Q \cup \bigcup_{i=1}^{n} S_{i}$ belong to $P(A ; \varepsilon, \not{H} ; \delta)$. Hence

$$
|\sigma(f, P)-\sigma(f, Q)|=\left|\sigma\left(f, P^{*}\right)-\sigma\left(f, Q^{*}\right)\right|<\varepsilon,
$$

and the proposition follows from 3.3.

3.5. LEMMA. Let $B$ and $D$ be overlapping intervals, and let $\forall$ be a finite family of planes such that $H \cap H^{*} \in \mathcal{H}$ for each $H, H^{*} \in \mathcal{H}$ with $H \cap H^{*} \neq \varnothing$. If $\partial D \subset \bigcup \not{H}$ and $D \cap H \neq \varnothing$ for each $H \in \mathcal{H}$, then $r(B ; \not{H}) \leq r(B \cap D ; \not{H})$.

ProOF. Let $\varkappa_{0}$ be the family of all planes in $\{H \in \not H: B \cap H \neq \varnothing\}$ which are minimal with respect to the inclusion. It follows from 2.1 that $r\left(B ; \mathscr{H}_{0}\right)=r(B ; \not{K})$. Let $B=\prod_{i=1}^{m}\left[a_{i}, b_{i}\right], D=\prod_{i=1}^{m}\left[\alpha_{i}, \beta_{i}\right]$, and let $H \in \mathscr{H}_{0}$. By symmetry, we may assume that

$$
H=\left\{\left(\xi_{1}, \ldots, \xi_{m}\right) \in \mathbf{R}^{m}: \xi_{i}=c_{i}, i=1, \ldots, k\right\}
$$

where $1 \leq k \leq m$ and $c_{i} \in\left[a_{i}, b_{i}\right] \cap\left[\alpha_{i}, \beta_{i}\right], i=1, \ldots, k$. Suppose there is a $y=\left(c_{1}, \ldots, c_{k}, \eta_{k+1}, \ldots, \eta_{m}\right)$ in $(B-D) \cap H$. Then $k<m$ and $\eta_{j} \in\left[a_{j}, b_{j}\right]-\left[\alpha_{j}, \beta_{j}\right]$ for some $j>k$. With no loss of generality, we assume that $\eta_{j}<\alpha_{j}$. As $\not k$ is finite and $\partial D \subset \bigcup \nvdash$, the plane $H^{*}=\left\{\left(\xi_{1}, \ldots, \xi_{m}\right) \in \mathbf{R}^{m}: \xi_{j}=\alpha_{j}\right\}$ belongs to $\mathscr{H}$, and since $H \cap H^{*} \neq \varnothing$, so does $H \cap H^{*}$. Now $H \cap H^{*} \subsetneq H$ and the point $\left(c_{1}, \ldots, c_{k}, a_{k+1}, \ldots, a_{j-1}, \alpha_{j}, a_{j+1}, \ldots, a_{m}\right)$ belongs to $B \cap H \cap H^{*} ;$ for $a_{j} \leq \eta_{j}<$ $\alpha_{j}$ and $B^{\circ} \cap D^{\circ} \neq \varnothing$ implies $\alpha_{j}<b_{j}$. This contradicts the minimality of $H$, and we conclude that $B \cap H=B \cap D \cap H$. Consequently, $r\left(B ; \not{H} \mathscr{H}_{0}\right) \leq r\left(B \cap D ; \not{H}{ }_{0}\right) \leq$ $r(B \cap D ; H)$. 
3.6. Proposition. Let $f$ be a function on an interval $A$, and let $D$ be a division of $A$. If $f \in R(D)$ for each $D \in D$, then $f \in R(A)$ and $\int_{A} f=\sum_{D \in D} \int_{D} f$.

Proof. Choose a regulator $(\varepsilon, \mathcal{H})$ so that $\bigcup_{D \in D} \partial D \subset \bigcup \mathcal{H}$ and $H \cap H^{*} \in \mathcal{H}$ for each $H, H^{*} \in \mathcal{H}$ with $H \cap H^{*} \neq \varnothing$. Let $\eta: A \rightarrow \mathbf{R}_{+}$be such that $\eta(x)<\operatorname{dist}(x, H)$ for each $H \in \forall$ and for each $x \in A-H$. In particular, if $D \in D$ and $x \in A-D$, then $\eta(x)<\operatorname{dist}(x, D)$; for $\partial D \subset \bigcup H$. Now given $D \in D$, find a $\delta_{D}: D \rightarrow \mathbf{R}_{+}$ so that $\delta_{D} \leq \eta$ and $\left|\sigma(f, P)-\int_{D} f\right|<\varepsilon|D| /|A|$ for each $P \in \mathcal{P}\left(D ; \varepsilon, \not H ; \delta_{D}\right)$. For $x \in A$ set

$$
\delta(x)=\min \left\{\delta_{D}(x): D \in D, x \in D\right\},
$$

and select a $P \in P(A ; \varepsilon, \mathscr{H} ; \delta)$. Let $(B, x) \in P, D \in D$, and $B^{\circ} \cap D^{\circ} \neq \varnothing$. Since $\delta \leq \eta$, we see that $x \in D$ and $B \cap H=\varnothing$ for each $H \in \mathcal{H}$ with $x \notin H$. Thus $B$ meets only those planes from $\sharp$ which meet $D$, and so by 2.1(i) and 3.5, $r(B \cap D ; \not{H}) \geq r(B ; \not{H}) \geq \varepsilon$. It follows that

$$
P_{D}=\left\{(B \cap D, x):(B, x) \in P, B^{\circ} \cap D^{\circ} \neq \varnothing\right\}
$$

belongs to $\mathcal{P}\left(D ; \varepsilon, \not H ; \delta_{D}\right)$ for each $D \in D$. As $\sigma(f, P)=\sum_{D \in D} \sigma\left(f, P_{D}\right)$, we have

$$
\left|\sigma(f, P)-\sum_{D \in D} \int_{D} f\right| \leq \sum_{D \in D}\left|\sigma\left(f, P_{D}\right)-\int_{D} f\right|<\frac{\varepsilon}{|A|} \sum_{D \in D}|D|=\varepsilon .
$$

4. The relationship to the Lebesgue integral, and its consequences. If $E \subset \mathbf{R}^{m}$ is measurable, we denote by $\mathcal{L}(E)$ the family of all functions $f$ on $E$ for which the finite Lebesgue integral (L) $\int_{E} f$ exists.

4.1. Proposition. Let $A$ be an interval. Then $\mathcal{L}(A) \subset R(A)$ and $\int_{A} f=$ (L) $\int_{A} f$ for each $f \in \mathcal{L}(A)$.

Proof. Let $f \in \mathcal{L}(A)$ and $\varepsilon>0$. There are functions $g$ and $h$ on $A$ which are, respectively, upper and lower semicontinuous, and such that $g \leq f \leq h$ and $(\mathrm{L}) \int_{A}(h-g)<\varepsilon / 2$. Find a $\delta: A \rightarrow \mathbf{R}_{+}$so that $g(y) \leq g(x)+\varepsilon / 2|A|$ and $h(y) \geq h(x)-\varepsilon / 2|A|$ for each $x, y \in A$ with $|x-y|<\delta(x)$. Now let $P=\left\{\left(A_{1}, x_{1}\right), \ldots,\left(A_{p}, x_{p}\right)\right\}$ be a $\delta$-fine partition of $A$. By our choice of $\delta$ we have

$$
\text { (L) } \begin{aligned}
\int_{A_{i}} g-\frac{\varepsilon\left|A_{i}\right|}{2|A|} & \leq g\left(x_{i}\right)\left|A_{i}\right| \leq f\left(x_{i}\right)\left|A_{i}\right| \leq h\left(x_{i}\right)\left|A_{i}\right| \\
& \leq(\mathrm{L}) \int_{A_{i}} h+\frac{\varepsilon\left|A_{i}\right|}{2|A|}
\end{aligned}
$$

and since $(\mathrm{L}) \int_{A_{i}} g \leq(\mathrm{L}) \int_{A_{i}} f \leq(\mathrm{L}) \int_{A_{i}} h$, we obtain

$$
\left|f\left(x_{i}\right)\right| A_{i}\left|-(\mathrm{L}) \int_{A_{i}} f\right| \leq \frac{\varepsilon\left|A_{i}\right|}{2|A|}+(\mathrm{L}) \int_{A_{i}}(h-g),
$$

$i=1, \ldots, p$. Consequently,

$$
\begin{aligned}
\left|\sigma(f, P)-(\mathrm{L}) \int_{A} f\right| & \leq \sum_{i=1}^{p}\left|f\left(x_{i}\right)\right| A_{i}\left|-(\mathrm{L}) \int_{A_{i}} f\right| \\
& \leq \frac{\varepsilon}{2}+(\mathrm{L}) \int_{A}(h-g)<\varepsilon .
\end{aligned}
$$


We conclude that $f \in R(A)$ and $\int_{A} f=(\mathrm{L}) \int_{A} f$.

4.2. COROLLARY. Let $f$ and $g$ be functions on an interval $A$, and let $f(x)=$ $g(x)$ for almost all $x \in A$. Then $f \in R(A)$ if and only if $g \in R(A)$, in which case $\int_{A} f=\int_{A} g$.

As $f-g$ is in $\mathcal{L}(A)$, the corollary follows from 4.1 and 3.2.

4.3. LEMMA. Let $f$ be a function integrable on an interval $A$, and let $(\varepsilon, H)$ be a regulator. Then there is a $\delta: A \rightarrow \mathbf{R}_{+}$such that

$$
\sum_{i=1}^{p}\left|f\left(x_{i}\right)\right| A_{i}\left|-\int_{A_{i}} f\right|<\varepsilon
$$

for each $\left\{\left(A_{1}, x_{1}\right), \ldots,\left(A_{p}, x_{p}\right)\right\}$ in $\mathcal{P}(A ; \varepsilon, \mathscr{H} ; \delta)$.

Proof. Find a $\delta: A \rightarrow \mathbf{R}_{+}$so that $\left|\sigma(f, P)-\int_{A} f\right|<\varepsilon / 3$ for each $P \in$ $\mathcal{P}(A ; \varepsilon, \mathcal{H} ; \delta)$, and choose a partition $\left\{\left(A_{1}, x_{1}\right), \ldots,\left(A_{p}, x_{p}\right)\right\}$ in $\mathcal{P}(A ; \varepsilon, \mathscr{H} ; \delta)$. We may assume that $f\left(x_{i}\right)\left|A_{i}\right| \geq \int_{A_{i}} f$ for $i=1, \ldots, k$, and $f\left(x_{i}\right)\left|A_{i}\right|<\int_{A_{i}} f$ for $i=k+1, \ldots, p$, where $0 \leq k \leq p$. By 3.4 and 2.5 , there are $P_{i} \in \mathcal{P}\left(A_{i} ; \varepsilon, \not{K} ; \delta\right)$ such that $\left|\sigma\left(f, P_{i}\right)-\int_{A_{i}} f\right|<\varepsilon / 3 p, i=1, \ldots, p$. Since

$$
\begin{aligned}
& P_{+}=\left\{\left(A_{1}, x_{1}\right), \ldots,\left(A_{k}, x_{k}\right)\right\} \cup \bigcup_{i=k+1}^{p} P_{i}, \\
& P_{-}=\left\{\left(A_{k+1}, x_{k+1}\right), \ldots,\left(A_{p}, x_{p}\right)\right\} \cup \bigcup_{i=1}^{k} P_{i}
\end{aligned}
$$

belong to $\mathcal{P}(A ; \varepsilon, \not H ; \delta)$, we have

$$
\begin{aligned}
\frac{\varepsilon}{3} & >\sigma\left(f, P_{+}\right)-\int_{A} f=\sum_{i=1}^{k}\left|f\left(x_{i}\right)\right| A_{i}\left|-\int_{A_{i}} f\right|+\sum_{i=k+1}^{p}\left[\sigma\left(f, P_{i}\right)-\int_{A_{i}} f\right] \\
& >\sum_{i=1}^{k}\left|f\left(x_{i}\right)\right| A_{i}\left|-\int_{A_{i}} f\right|-\frac{\varepsilon(p-k)}{3 p}
\end{aligned}
$$

and similarly, using $P_{-}$, we also have

$$
\frac{\varepsilon}{3}>\sum_{i=k+1}^{p}\left|f\left(x_{i}\right)\right| A_{i}\left|-\int_{A_{i}} f\right|-\frac{\varepsilon k}{3 p} .
$$

Therefore

$$
\sum_{i=1}^{p}\left|f\left(x_{i}\right)\right| A_{i}\left|-\int_{A_{i}} f\right|<\varepsilon
$$

Let $F$ be a function of subintervals of an interval $A$, and let $x \in A$. Following [S, Chapter 4, §2], we say that $F$ is derivable at $x$ if a finite $\lim \left[F\left(B_{n}\right) /\left|B_{n}\right|\right]$ exists for each sequence $\left\{B_{n}\right\}$ of subintervals of $A$ such that $x \in B_{n}, n=1,2, \ldots$, $\lim d\left(B_{n}\right)=0$, and $\inf r\left(B_{n}\right)>0$. If all these limits exist, then they have the same value, denoted by $F^{\prime}(x)$ and called the derivative of $F$ at $x$. 
4.4. Proposition. Let $f$ be an integrable function on an interval $A$, and let $F(B)=\int_{B} f$ for each interval $B \subset A$. Then for almost all $x \in A$ the function $F$ is derivable at $x$ and $F^{\prime}(x)=f(x)$.

ProOF. We employ the technique introduced in [LW]. Let $E$ be the set of all $x \in A$ such that either $F$ is not derivable at $x$, or $F^{\prime}(x) \neq f(x)$. It follows directly from the definition of $F^{\prime}(x)$ that given $x \in E$, we can find an $\alpha(x)>0$ such that for each $\beta>0$ there is an interval $B \subset A$ with $x \in B, d(B)<\beta, r(B) \geq \alpha(x)$, and $|[F(B) /|B|]-f(x)| \geq \alpha(x)$. Fix an integer $n \geq 2$, and let $E_{n}=\{x \in E: \alpha(x)>$ $1 / n\}$. Choose an $\varepsilon \in(0,1 / n]$ and use 4.3 to find a $\delta: A \rightarrow \mathbf{R}_{+}$, such that

$$
\sum_{i=1}^{p}\left|f\left(x_{i}\right)\right| A_{i}\left|-F\left(A_{i}\right)\right|<\frac{\varepsilon}{n}
$$

for each $\left\{\left(A_{1}, x_{1}\right), \ldots,\left(A_{p}, x_{p}\right)\right\}$ in $P(A ; \varepsilon, \varnothing ; \delta)$. Let $B$ be the family of all intervals $B \subset A$ such that $r(B) \geq \varepsilon, d(B)<\delta\left(x_{B}\right)$ for some $x_{B} \in B \cap E_{n}$, and $\left|f\left(x_{B}\right)\right| B \mid-$ $F(B)|\geq| B \mid / n$. It is easy to verify that $B$ covers $E_{n}$ in the sense of Vitali. Thus by $\left[\mathrm{S}\right.$, Chapter 4, Theorem (3.1)], there are disjoint intervals $B_{1}, B_{2}, \ldots$ in $B$ such that $\left|E_{n}-\bigcup_{k=1}^{\infty} B_{k}\right|=0$. Using 2.5 , it is easy to see that each $\left\{\left(B_{1}, x_{B_{1}}\right), \ldots,\left(B_{s}, x_{B_{s}}\right)\right\}$ is a subset of some $P \in P(A ; \varepsilon, \varnothing ; \delta)$. Thus

$$
\sum_{k=1}^{s}\left|B_{k}\right| \leq n \sum_{k=1}^{s}\left|f\left(x_{B_{k}}\right)\right| B_{k}\left|-F\left(B_{k}\right)\right|<\varepsilon
$$

for $s=1,2, \ldots$, and consequently

$$
\left|E_{n}\right|=\left|E_{n} \cap \bigcup_{k=1}^{\infty} B_{k}\right| \leq \sum_{k=1}^{\infty}\left|B_{k}\right|<\varepsilon .
$$

The arbitrariness of $\varepsilon$ implies that $\left|E_{n}\right|=0$. As $E=\bigcup_{n=2}^{\infty} E_{n}$, we also have $|E|=0$.

4.5. COROLlaRY. Each integrable function on an interval $A$ is measurable.

The corollary follows directly from 4.4 and [S, Chapter 4, Theorem (4.2)].

4.6. Proposition. Let $f$ be a function on an interval $A$. Then $f$ belongs to $\mathcal{L}(A)$ if and only if both $f$ and $|f|$ belong to $R(A)$.

Proof. If $f$ and $|f|$ are integrable, then by $4.5,|f|$ is Lebesgue integrable. Moreover, by 4.1 and 3.2 ,

$$
\text { (L) } \int_{A}|f|=\lim (\mathrm{L}) \int_{A} \min (|f|, n)=\lim \int_{A} \min (|f|, n) \leq \int_{A}|f|<+\infty,
$$

and so $|f| \in \mathcal{L}(A)$. From this and 4.5, we see that $f \in \mathcal{L}(A)$. The converse follows from 4.1.

4.7. COROLlaRY. Let $f$ be an integrable function on an interval $A$. If $\int_{B} f=0$ for each interval $B \subset A$, then $f(x)=0$ for almost all $x \in A$.

It follows from 4.3 that $|f| \in R(A)$ and $\int_{A}|f|=0$. Hence the corollary is a consequence of 4.6 and 4.1 . 
4.8. COROLLARY. Let $\left\{f_{n}\right\}$ be an increasing sequence of integrable functions on an interval $A$, and let $\lim \int_{A} f_{n}<+\infty$. If $f=\lim f_{n}$, then $f \in R(A)$ and $\int_{A} f=\lim \int_{A} f_{n}$.

Using 3.2 and 4.6, we can apply the monotone convergence theorem for the Lebesgue integral to the sequence $\left\{f_{n}-f_{1}\right\}$. Then the corollary follows from 4.1 and 3.2.

4.9. COROLLARY. Let $f_{n}, g$, and $h$ be integrable functions on an interval $A$, and let $g \leq f_{n} \leq h, n=1,2, \ldots$. If $f=\lim f_{n}$, then $f \in R(A)$ and $\int_{A} f=$ $\lim \int_{A} f_{n}$.

Using 4.6, 4.1, and 3.2, it suffices to apply the dominated convergence theorem for the Lebesgue integral to the sequence $\left\{f_{n}-g\right\}$.

We say that a sequence $\left\{B_{n}\right\}$ of intervals converges to a set $E \subset \mathbf{R}^{m}$ whenever $E \subset B_{n}, n=1,2, \ldots$, and each open set $G \subset \mathbf{R}^{m}$ containing $E$ contains $B_{n}$ for all sufficiently large $n$ 's.

4.10. Proposition. Let $f$ be an integrable function on an interval $A$, let $B \subset A$ be an interval, and let $H$ be a plane with $B \cap H \neq \varnothing$. If a sequence $\left\{B_{n}\right\}$ of subintervals of $A$ converges to $B \cap H$, then $\lim \int_{B_{n}} f=0$.

PROOF. In view of 4.2, we may assume that $f(x)=0$ for every $x \in B \cap H$. Choose a regulator $(\varepsilon, \mathcal{E})$ so that $H \in \mathcal{E}, \partial B \subset \cup \mathcal{E}$, and $E \cap E^{*} \in \mathcal{E}$ for each $E, E^{*} \in \mathcal{E}$ with $E \cap E^{*} \neq \varnothing$. By 4.3 , there is a $\delta: A \rightarrow \mathbf{R}_{+}$such that

$$
\sum_{i=1}^{p}\left|f\left(x_{i}\right)\right| A_{i}\left|-\int_{A_{i}} f\right|<\varepsilon
$$

for every $\left\{\left(A_{1}, x_{1}\right), \ldots,\left(A_{p}, x_{p}\right)\right\}$ in $\mathcal{P}(A ; \varepsilon, \mathcal{E} ; \delta)$. With no loss of generality, we can assume that $\delta(x)<\operatorname{dist}(x, E)$ for each $E \in \mathcal{E}$ and for each $x \in A-E$. Now given a sequence $\left\{B_{n}\right\}$ of subintervals of $A$ converging to $B \cap H$, we consider two cases.

(i) Let $H=\{x\}$ be a 0-plane, and let $G=\left\{y \in \mathbf{R}^{m}:|x-y|<\delta(x)\right\}$. If $B_{n} \subset G$, then $\left\{\left(B_{n}, x\right)\right\}$ is a subset of some $P \in P(A ; \varepsilon, \mathcal{E} ; \delta)$, and we have $\left|\int_{B_{n}} f\right|<\varepsilon$.

(ii) Let $H$ be a $k$-plane with $k \geq 1$. Using 2.4 for $m=k$, we can find a $\delta$-fine $(\varepsilon, \varnothing)$-partition $\left\{\left(C_{1}, x_{1}\right), \ldots,\left(C_{s}, x_{s}\right)\right\}$ of the $k$-dimensional interval $B \cap H$. Let $\eta=\min \left\{\delta\left(x_{1}\right), \ldots, \delta\left(x_{s}\right)\right\}$ and

$$
G=\left\{y \in \mathbf{R}^{m}: \operatorname{dist}(y, B \cap H)<\eta\right\} .
$$

If $B_{n} \subset G$, then using our choice of $\delta$, it is easy to construct a $\delta$-fine partition $Q=$ $\left\{\left(D_{1}, y_{1}\right), \ldots,\left(D_{q}, y_{q}\right)\right\}$ of $B_{n}$ so that $\left\{y_{1}, \ldots, y_{q}\right\} \subset\left\{x_{1}, \ldots, x_{s}\right\}$, and $D_{j} \cap B \cap H$ is a face of $C_{i}$ whenever $y_{j}=x_{i}$. From the choice of $\mathcal{E}$ it is not difficult to deduce that $Q \in P\left(B_{n} ; \varepsilon, \mathcal{E} ; \delta\right)$. Thus by $2.4, Q$ is a subset of a $P \in P(A ; \varepsilon, \mathcal{E} ; \delta)$, and we have

$$
\left|\int_{B_{n}} f\right| \leq \sum_{j=1}^{q}\left|\int_{D_{j}} f\right|<\varepsilon .
$$

The next corollary follows from Propositions 3.4, 3.6, and 4.10. We omit its tedious but straightforward proof. 
4.11. COROLlaRY. Let $f$ be an integrable function on an interval $A$, and let $\left\{B_{n}\right\}$ be a sequence of subintervals of $A$ such that $\bigcup_{k=1}^{\infty} \bigcap_{n=k}^{\infty} B_{n}=\bigcap_{k=1}^{\infty} \bigcup_{n=k}^{\infty} B_{n}$. If $B$ is the closure of $\bigcup_{k=1}^{\infty} \bigcap_{n=k}^{\infty} B_{n}$, then $\lim \int_{B_{n}} f=\int_{B}^{n} f$ if $B$ is an interval, and $\lim \int_{B_{n}} f=0$ otherwise.

5. The divergence theorem. Throughout this section, we assume that $m \geq 2$. By $x \cdot y$ we denote the usual inner product of $x, y \in \mathbf{R}^{m}$, and we set $\|x\|=\sqrt{x \cdot x}$. However, notwithstanding the introduction of the Euclidean norm $\|x\|$, the metric in $\mathbf{R}^{m}$ is still that induced by the norm $|x|$.

If $H$ is a $k$-plane, $k \geq 1$, and $E \subset H$ is measurable with respect to the $k$ dimensional Lebesgue measure in $H$, we denote by $\left(\mathrm{L}_{k}\right) \int_{E} f$ the $k$-dimensional Lebesgue integral of $f: E \rightarrow \mathbf{R}$ (provided it exists). If $A$ is an interval, then using the $(m-1)$-dimensional Lebesgue integral, we can define the flux of a vector field $v: \partial A \rightarrow \mathbf{R}^{m}$ across $\partial A$ in the direction of the exterior normal $n$ as the surface integral $\left(\mathrm{L}_{m-1}\right) \int_{\partial A} v \cdot n$ (provided it exists).

The word differentiable is used in the sense of [R, Definition 9.11]. Thus differentiability of a function implies its continuity and the existence of finite partial derivatives, which need not be continuous. The $i$ th partial derivative of a differentiable function $f$ is denoted by $\partial_{i} f, i=1, \ldots, m$. If $v=\left(f_{1}, \ldots, f_{m}\right)$ is a vector field on an interval $A$, we call a divergence of $v$ any function $g$ on $A$ such that $g(x)=\sum_{i=1}^{m} \partial_{i} f_{i}(x)$ for each $x \in A^{\circ}$ at which $v$ is differentiable. Each divergence of a vector field $v$ is denoted by $\nabla \cdot v$.

In the following three lemmas we assume that $A$ is an interval, and that $v: A \rightarrow$ $\mathbf{R}^{m}$ is a vector field such that a finite $\left(\mathrm{L}_{m-1}\right) \int_{\partial B} v \cdot n$ exists for each interval $B \subset A$.

5.1. LemMA. Let $v$ be differentiable at $x \in A^{\circ}$. Then given $\varepsilon>0$, there is a $\delta>0$ such that

$$
|\nabla \cdot v(x)| B\left|-\left(\mathrm{L}_{m-1}\right) \int_{\partial B} v \cdot n\right|<\varepsilon|B|
$$

for each interval $B \subset A$ with $x \in B, d(B)<\delta$, and $r(B) \geq \varepsilon$.

PROOF. It suffices to prove the lemma for $v=(f, 0, \ldots, 0)$. Let $x=\left(\xi_{1}, \ldots, \xi_{m}\right)$ and $y=\left(\eta_{1}, \ldots, \eta_{m}\right)$ be in $A$. By our assumption there is a function $\omega$ on $A$ such that $\lim _{y \rightarrow x} \omega(y)=0$ and

$$
f(y)-f(x)=\sum_{i=1}^{m}\left[\partial_{i} f(x)+\omega(y)\right]\left(\eta_{i}-\xi_{i}\right) .
$$

Now given $\varepsilon>0$, find $\delta>0$ so that $|\omega(y)|<\varepsilon^{2} / 2 m$ whenever $|y-x|<\delta$. Let $B=[a, b] \times C$ be a subinterval of $A$ such that $x \in B, d=d(B)<\delta$, and $r(B) \geq \varepsilon$. For any $t=\left(\tau_{2}, \ldots, \tau_{m}\right)$ in $C$, set $f^{a}(t)=f(a, t)$ and $f^{b}(t)=f(b, t)$. Then

$$
\begin{aligned}
f^{b}(t)-f^{a}(t)= & {[f(b, t)-f(x)]+[f(x)-f(a, t)] } \\
= & \partial_{1} f(x)(b-a)+\omega(b, t)\left(b-\xi_{1}\right)+\omega(a, t)\left(\xi_{1}-a\right) \\
& +[\omega(b, t)-\omega(a, t)] \sum_{i=2}^{m}\left(\tau_{i}-\xi_{i}\right),
\end{aligned}
$$


and so

From this we obtain

$$
\begin{aligned}
\left|f^{b}-f^{a}-\partial_{1} f(x)(b-a)\right| & <\frac{\varepsilon^{2}}{2 m}(b-a)+\frac{\varepsilon^{2}}{m} \sum_{i=2}^{m}\left|\tau_{i}-\xi_{i}\right| \\
& \leq \frac{\varepsilon^{2}}{m} d\left[\frac{1}{2}+(m-1)\right]<\varepsilon^{2} d .
\end{aligned}
$$

$$
\begin{aligned}
& \left|\left(\mathrm{L}_{m-1}\right) \int_{\partial B} v \cdot n-\nabla \cdot v(x)\right| B|| \\
& \quad=\left|\left(\mathrm{L}_{m-1}\right) \int_{C}\left[f^{b}-f^{a}\right]-\left(\mathrm{L}_{m-1}\right) \int_{C} \partial_{1} f(x)(b-a)\right| \\
& \quad<\varepsilon^{2} d|C|_{m-1} \leq \varepsilon^{2} d^{m}=\varepsilon^{2} \frac{|B|}{r(B)} \leq \varepsilon|B| .
\end{aligned}
$$

5.2. LEMMA. Let $v$ be continuous at $x \in A$, and let $H$ be an $(m-1)$-plane containing $x$. Then given $\varepsilon>0$, there is a $\delta>0$ such that

$$
\left|\left(\mathrm{L}_{m-1}\right) \int_{\partial B} v \cdot n\right|<\varepsilon|B \cap H|_{m-1}
$$

for each interval $B \subset A$ with $x \in B, d(B)<\delta$, and $r(B ;\{H\}) \geq \varepsilon$.

ProOF. Given $\varepsilon>0$, find a $\delta>0$, so that $\|v(y)-v(x)\|<\varepsilon^{2} / 2 m$ whenever $y \in A$ and $|y-x|<\delta$. Now if $B$ is a subinterval of $A, x \in B, d(B)<\delta$, and $r(B ;\{H\}) \geq \varepsilon$, then

$$
\begin{aligned}
\left|\left(\mathrm{L}_{m-1}\right) \int_{\partial B} v \cdot n\right| & =\left|\left(\mathrm{L}_{m-1}\right) \int_{\partial B}[v-v(x)] \cdot n\right|<\frac{\varepsilon^{2}}{2 m}|\partial B|_{m-1} \\
& \leq \varepsilon^{2}[d(B)]^{m-1}=\varepsilon^{2} \frac{|B \cap H|_{m-1}}{r(B ;\{H\})} \leq \varepsilon|B \cap H|_{m-1} .
\end{aligned}
$$

5.3. LEMMA. Let $k$ be an integer, $0 \leq k \leq m-2$, and let $\alpha: \mathbf{R}_{+} \rightarrow \mathbf{R}_{+}$be a decreasing function with $\left(\mathrm{L}_{1}\right) \int_{0}^{1} \alpha<+\infty$. Further, let $H$ be a $k$-plane containing $a$ point $x \in A$, and suppose that

$$
\|v(y)-v(x)\|=O\left[\alpha(\|y-x\|)\|y-x\|^{k+2-m}\right]
$$

as $y$ approaches $x$. Then given $\varepsilon>0$, there is a $\delta>0$ such that

$$
\left|\left(\mathrm{L}_{m-1}\right) \int_{\partial B} v \cdot n\right|<\varepsilon|B \cap H|_{k}
$$

for each interval $B \subset A$ with $x \in B, d(B)<\delta$, and $r(B ;\{H\}) \geq \varepsilon$.

ProOF. Given $\varepsilon>0$, there are $M>0$ and $\eta>0$ such that

$$
\|v(y)-v(x)\| \leq M \alpha(\|y-x\|)\|y-x\|^{k+2-m}
$$

for each $y \in A$ with $0<|y-x|<\eta$. Set $N=4 \pi^{m-2} m^{(k / 2)+1} M$, and find $\delta>0$ so that $\delta \leq \eta$ and $\left(\mathrm{L}_{1}\right) \int_{0}^{\delta \sqrt{m}} \alpha<\varepsilon^{2} / N$. Let $B$ be a subinterval of $A, x \in B$, $d=d(B)<\delta$, and $r(B ;\{H\}) \geq \varepsilon$. If $\Omega=\left\{y \in \mathbf{R}^{m}:\|x-y\| \leq d \sqrt{m}\right\}$ and $E$ is an $(m-1)$-plane containing $x$, then the integral

$$
I=\left(\mathrm{L}_{m-1}\right) \int_{E \cap \Omega} \alpha(\|y-x\|)\|y-x\|^{k+2-m} d y
$$


is independent of the choice of $E$; for both $\Omega$ and the integrand are invariant with respect to rotations about $x$. Since the integrand in $I$ is a decreasing function of $\|y-x\|$, we have

$$
\begin{aligned}
\left|\left(\mathrm{L}_{m-1}\right) \int_{\partial B} v \cdot n\right| & =\left|\left(\mathrm{L}_{m-1}\right) \int_{\partial B}[v-v(x)] \cdot n\right| \\
& \leq M\left(\mathrm{~L}_{m-1}\right) \int_{\partial B} \alpha(\|y-x\|)\|y-x\|^{k+2-m} d y \leq 2 m M I .
\end{aligned}
$$

Changing to spherical coordinates about $x$ in $E$, we see that

$$
\begin{aligned}
I & \leq 2 \pi^{m-2}\left(\mathrm{~L}_{1}\right) \int_{0}^{d \sqrt{m}} \alpha(t) t^{k} d t \\
& \leq 2 \pi^{m-2}(d \sqrt{m})^{k}\left(\mathrm{~L}_{1}\right) \int_{0}^{d \sqrt{m}} \alpha<\frac{\varepsilon^{2}}{2 m M} d^{k},
\end{aligned}
$$

and hence

$$
\left|\left(\mathrm{L}_{m-1}\right) \int_{\partial B} v \cdot n\right|<\varepsilon^{2} d^{k}=\varepsilon^{2} \frac{|B \cap H|_{k}}{r(B ;\{H\})} \leq \varepsilon|B \cap H|_{k} .
$$

We are now in a position to prove the divergence theorem.

5.4. THEOREM. Let $S_{-1}=\varnothing$, and for $k=0, \ldots, m-1$, let $S_{k}$ be a countable union of $k$-planes. Let $v$ be a vector field on an interval $A$, and suppose that the following conditions are satisfied:

(i) $v$ is differentiable in $A^{\circ}-S_{m-1}$;

(ii) $v$ is continuous in $A-S_{m-2}$;

(iii) for each $x \in A-S_{k}, k=-1, \ldots, m-3$, there is a decreasing function $\alpha_{x}: \mathbf{R}_{+} \rightarrow \mathbf{R}_{+}$such that $\left(\mathrm{L}_{1}\right) \int_{0}^{1} \alpha_{x}<+\infty$ and

$$
\|v(y)-v(x)\|=O\left[\alpha_{x}(\|y-x\|)\|y-x\|^{k+3-m}\right]
$$

as $y$ approaches $x$.

Then $\left(\mathrm{L}_{m-1}\right) \int_{\partial A} v \cdot n$ exists, $\nabla \cdot v \in R(A)$, and $\int_{A} \nabla \cdot v=\int_{\partial A} v \cdot n$.

PROOF. Let $H$ be an $(m-1)$-plane and let $x \in A \cap H$. By (ii), the vector field $v$ on $A \cap H$ is measurable with respect to the $(m-1)$-dimensional Lebesgue measure in $H$; for $\left|H \cap S_{m-2}\right|_{m-1}=0$. By (iii), there exist $M>0$ and $\beta \in(0,1]$ such that

$$
\begin{aligned}
\|v(y)\| & \leq\|v(x)\|+\|v(y)-v(x)\| \\
& \leq\|v(x)\|+M \alpha_{x}(\|y-x\|)\|y-x\|^{2-m}
\end{aligned}
$$

for each $y \in A$ with $0<\|y-x\|<\beta$. If $\Omega=\{y \in H:\|y-x\|<\beta\}$, then using spherical coordinates about $x$ in $H$, we see that

$$
\left(\mathrm{L}_{m-1}\right) \int_{\Omega} \alpha_{x}(\|y-x\|)\|y-x\|^{2-m} d y \leq 2 \pi^{m-2}\left(\mathrm{~L}_{1}\right) \int_{0}^{1} \alpha_{x}<+\infty .
$$

It follows that $\left(\mathrm{L}_{m-1}\right) \int_{A \cap \Omega}\|v\|<+\infty$, and since $A \cap H$ is compact, we also have $\int_{A \cap H}\|v\|<+\infty$. Thus a finite $\left(\mathrm{L}_{m-1}\right) \int_{\partial B} v \cdot n$ exists for each interval $B \subset A$, and the common assumption of Lemmas 5.1-5.3 is satisfied. In particular, $\left(\mathrm{L}_{m-1}\right) \int_{\partial A} v \cdot n$ exists and it is finite. 
Choose a regulator $(\varepsilon, \mathcal{H})$ so that $\partial A \subset \bigcup \mathcal{H}$ and $H \cap H^{*} \in \mathcal{H}$ for each $H, H^{*} \in \mathcal{H}$ with $H \cap H^{*} \neq \varnothing$. For $k=0, \ldots, m-1$, let $S_{k}=\left\{H_{k}^{1}, H_{k}^{2}, \ldots\right\}$ be a countable family of $k$-planes whose union is $S_{k}$, and let $S=\bigcup_{k=0}^{m-1} S_{k}$. Replacing the $S_{k}$ 's by larger countable families of $k$-planes, we may assume that the following conditions hold:

(a) $H \subset S$;

(b) $S_{k-1} \subset S_{k}, k=0, \ldots, m-1$;

(c) $H \cap H^{*} \in S$ for each $H, H^{*} \in S$ with $H \cap H^{*} \neq \varnothing$.

There exists a $\delta: A \rightarrow(0,1]$ having the following properties:

(d) $\delta(x)<\operatorname{dist}(x, H)$ for each $H \in \forall$ and for each $x \in A-H$;

(e) if $x \in A^{\circ}-S_{m-1}$, then

$$
|\nabla \cdot v(x)| B\left|-\left(\mathrm{L}_{m-1}\right) \int_{\partial B} v \cdot n\right|<\varepsilon|B|
$$

for each interval $B \subset A$ with $x \in B, d(B)<\delta(x)$, and $r(B) \geq \varepsilon$ (see 5.1);

(f) if $x \in H_{k}^{n}-S_{k-1}, k=0, \ldots, m-1$, then $\delta(x)<\varepsilon 2^{-n-1} /[|\nabla \cdot v(x)|+1]$ and

$$
\left|\left(\mathrm{L}_{m-1}\right) \int_{\partial B} v \cdot n\right|<\varepsilon 2^{-n-1}\left|B \cap H_{k}^{n}\right|_{k}
$$

for each interval $B \subset A$ with $x \in B, d(B)<\delta(x)$, and $r\left(B ;\left\{H_{k}^{n}\right\}\right) \geq \varepsilon$ (using 5.2 when $k=m-1$, and 5.3 otherwise). Now let $d=\max \{d(A), 1\}$, and select a $P=\left\{\left(A_{1}, x_{1}\right), \ldots,\left(A_{p}, x_{p}\right)\right\}$ in $\mathcal{P}(A ; \varepsilon, H ; \delta)$. We prove the theorem by showing that

To this end, let

$$
\left|\sigma(\nabla \cdot v, P)-\left(\mathrm{L}_{m-1}\right) \int_{\partial A} v \cdot n\right|<\varepsilon d^{m}(1+m) .
$$

$$
\alpha_{i}=\left|\nabla \cdot v\left(x_{i}\right)\right| A_{i}\left|-\left(\mathrm{L}_{m-1}\right) \int_{\partial A_{i}} v \cdot n\right|
$$

for $i=1, \ldots, p, X_{m}=\left\{x_{1}, \ldots, x_{p}\right\}-S_{m-1}$, and $X_{k}^{n}=\left\{x_{1}, \ldots, x_{p}\right\} \cap\left(H_{k}^{n}-S_{k-1}\right)$ for $k=0, \ldots, m-1$ and $n=1,2, \ldots$. If $x_{i} \in X_{m}$, then by (a), (b), and (d), $x_{i} \in A^{\circ}$ and $r\left(A_{i}\right)=r\left(A_{i} ; \mathcal{H}\right) \geq \varepsilon ;$ for $A_{i} \cap \bigcup \mathcal{H}=\varnothing$. Thus by (e),

$$
\sum_{x_{i} \in X_{m}} \alpha_{i}<\varepsilon \sum_{x_{i} \in X_{m}}\left|A_{i}\right| \leq \varepsilon|A| \leq \varepsilon d^{m} .
$$

Let $x_{i} \in X_{k}^{n}$. By (b), $x_{i}$ is contained in no plane from $\bigcup_{j=1}^{k-1} S_{j}$, and thus by (c), each plane from $\bigcup_{j=k}^{m-1} S_{j}$ containing $x_{i}$ contains $H_{k}^{n}$. In particular, each plane from $\mathcal{H}$ containing $x_{i}$ contains $H_{k}^{n}$. Choose an $H \in \mathcal{H}$ so that $r\left(A_{i} ;\{H\}\right)=r\left(A_{i} ; \mathcal{H}\right)$. If $A_{i} \cap H=\varnothing$, then by $2.1(\mathrm{i}), r\left(A_{i} ;\left\{H_{k}^{n}\right\}\right) \geq r\left(A_{i}\right)=r\left(A_{i} ;\{H\}\right)$. If $A_{i} \cap H \neq \varnothing$, then by 2.1(ii), again $r\left(A_{i} ;\left\{H_{k}^{n}\right\}\right) \geq r\left(A_{i} ;\{H\}\right)$; for by (d), $x \in H$ and hence $H_{k}^{n} \subset H$. Thus we always have $r\left(A_{i} ;\left\{H_{k}^{n}\right\}\right) \geq r(A ; \not{H}) \geq \varepsilon$, and applying (f), we obtain

$$
\begin{aligned}
\sum_{x_{i} \in X_{k}^{n}} \alpha_{i} & \leq \sum_{x_{i} \in X_{k}^{n}}\left\{\left|\nabla \cdot v\left(x_{i}\right)\right|\left|A_{i} \cap H_{k}^{n}\right|_{k}\left[d\left(A_{i}\right)\right]^{m-k}+\varepsilon 2^{-n-1}\left|A_{i} \cap H_{k}^{n}\right|_{k}\right\} \\
& \leq \varepsilon 2^{-n} \sum_{x_{i} \in X_{k}^{n}}\left|A_{i} \cap H_{k}^{n}\right|_{k} \leq \varepsilon 2^{-n}\left|A \cap H_{k}^{n}\right|_{k} \\
& \leq \varepsilon 2^{-n}[d(A)]^{k} \leq \varepsilon 2^{-n} d^{m}
\end{aligned}
$$


for $\left[d\left(A_{i}\right)\right]^{m-k} \leq\left[\delta\left(x_{i}\right)\right]^{m-k} \leq \delta\left(x_{i}\right)$. In conclusion,

$$
\begin{aligned}
\mid \sigma(\nabla & \cdot v, P)-\left(\mathrm{L}_{m-1}\right) \int_{\partial A} v \cdot n \mid \\
& \leq \sum_{i=1}^{p} \alpha_{i}=\sum_{x_{i} \in X_{m}} \alpha_{i}+\sum_{k=0}^{m-1} \sum_{n=1}^{\infty} \sum_{x_{i} \in X_{k}^{n}} \alpha_{i} \\
& <\varepsilon d^{m}\left(1+m \sum_{n=1}^{\infty} 2^{-n}\right)=\varepsilon d^{m}(1+m) .
\end{aligned}
$$

It appears worthwhile to formulate separately an important special case of Theorem 5.4 when $S_{0}=\cdots=S_{m-1}=\varnothing$.

5.5. COROLlARY. Let $v$ be a continuous vector field on the interval $A$ which is differentiable in $A^{\circ}$. Then $\nabla \cdot v \in R(A)$ and $\int_{A} \nabla \cdot v=\left(\mathrm{L}_{m-1}\right) \int_{\partial A} v \cdot n$.

5.6. REMARK. We proved Theorem 5.4 and Corollary 5.5 only for $m \geq 2$. However, their validity for $m=1$ is well known (see, e.g., [Mc, $§ 1.4$, p. 27]). It is also easy to see that, modulo some obvious adjustments, our proof applies to this much simpler case too.

5.7. EXAMPLE. Let $m=2$ and $I_{n}=\left[2^{-n-1}, 2^{-n}\right], n=0,1, \ldots$ Using a standard technique (see, e.g., [Mu, 1.3]), we construct continuously differentiable functions $\phi_{n}$ on $\mathbf{R}$ so that the following conditions are met:

(i) $0 \leq \phi_{n} \leq 1, \phi_{n}(t)=0$ for $t \leq(4 / 3) 2^{-n-1}$, and $\phi_{n}(t)=1$ for $t \geq(5 / 3) 2^{-n-1}$;

(ii) there is an $\alpha>0$ such that $\int_{I_{n}} \phi_{n} \geq \alpha 2^{-n}$ for $n=0,1, \ldots$ Given $(\xi, \eta)$ in $\mathbf{R}^{2}$, let $f(\xi, \eta)=0$ if $\eta \leq 0, f(\xi, \eta)=\eta^{2} \sin \xi$ if $\eta \geq 1$, and

$$
f(\xi, \eta)=\phi_{n}(\eta) \eta^{2} \sin 8^{n} \xi+\left[1-\phi_{n}(\eta)\right] \eta^{2} \sin 8^{n+1} \xi
$$

if $\eta \in I_{n}, n=0,1, \ldots$. In essence, $f$ is obtained by a smooth deformation of the function $\eta^{2} \sin 8^{n} \xi$ on the strip $\mathbf{R} \times\left[(5 / 3) 2^{-n-1},(4 / 3) 2^{-n}\right]$ to the function $\eta^{2} \sin 8^{n+1} \xi$ on the strip $\mathbf{R} \times\left[(5 / 3) 2^{-n-2},(4 / 3) 2^{-n-1}\right]$. It is easy to see that $f$ is differentiable in $\mathbf{R}^{2}$. Thus applying 5.5 to the vector field $v=(f, 0)$ in the interval $A=[0,2 \pi] \times[0,1]$, we see that $\partial_{1} f$ is integrable on $A$, and $\int_{A} \partial_{1} f=0$. However, we show that the integral $\int_{0}^{1} \partial_{1} f(\xi, \eta) d \eta$ does not exist for almost all $\xi \in[0,2 \pi]$, and hence the function $\partial_{1} f$ is not integrable on $A$ in the sense of [Mc] (see Chapter 6 , ibid.). Indeed, if $\int_{0}^{1} \partial_{1} f(\xi, \eta) d \eta$ exists, then by [Mc, Chapter 1, Ex. 5],

$$
\begin{aligned}
\int_{0}^{1} \partial_{1} f(\xi, \eta) d \eta=\sum_{n=0}^{\infty} \int_{I_{n}} \partial_{1} f(\xi, \eta) d \eta \\
\quad=\sum_{n=0}^{\infty}\left\{8^{n} \int_{I_{n}} \eta^{2} \phi_{n}(\eta) d \eta \cdot \cos 8^{n} \xi+8^{n+1} \int_{I_{n}} \eta^{2}\left[1-\phi_{n}(\eta)\right] d \eta \cdot \cos 8^{n+1} \xi\right\} .
\end{aligned}
$$

Since by (i) and (ii),

$$
\begin{aligned}
& 8^{n} \int_{I_{n}} \eta^{2} \phi_{n}(\eta) d \eta+8^{n+1} \int_{I_{n}} \eta^{2}\left[1-\phi_{n}(\eta)\right] d \eta \\
& \quad \geq 8^{n} \int_{I_{n}} \eta^{2} \phi_{n}(\eta) d \eta \geq 8^{n}\left(2^{-n-1}\right)^{2} \alpha 2^{-n}=\frac{\alpha}{4}
\end{aligned}
$$


$n=0,1, \ldots$, and since by $[\mathbf{K N}$, Chapter 1 , Theorem 4.1$]$, the sequence $\left\{8^{n} \xi\right\}$ is uniformly distributed $\bmod 2 \pi$ for almost all $\xi \in[0,2 \pi]$, it is easy to see that the above series diverges for almost all $\xi \in[0,2 \pi]$.

5.8. REMARK. The previous example shows that the Fubini theorem is false for the integral defined in 3.1. This is, of course, not a reparable deficiency of our definition, but rather the unavoidable price one must pay for making the divergence of each differentiable vector field integrable.

NoTE. The Fubini theorem is used in two principal ways: as a test for nonintegrability by showing that the iterated integrals have different values, and as a means of calculating integrals whose existence has been already established. For our integral, the former use is lost without any compensation; however, the loss of the latter deserves the following comment. Unlike for the Lebesgue integral, there are no easy existence criteria for nonabsolutely convergent integrals such as the Denjoy-Perron integral. Thus even when Fubini's theorem holds, we do not know a priori whether it can be applied. On the other hand, using the divergence theorem, we can often conveniently evaluate an integral as the flux of a suitably chosen vector field.

6. Change of variable. Let $\phi: A \rightarrow \mathbf{R}^{m}$ be a map of an interval $A$. We say that $\phi$ is admissible if it is continuous, and if there is a division $D$ of $A$ such that the restriction $\phi \uparrow D$ is affine for each $D \in D$. Clearly, among all such divisions of $A$ there is a unique division $D_{0}$ which is maximal with respect to refinements. For $D \in D_{0}$, the usual determinant of the affine map $\phi \uparrow D$ is $\operatorname{denoted}$ by $\operatorname{det}(\phi \uparrow D)$. Given $x \in A$, we set $(\operatorname{det} \phi)(x)=\operatorname{det}(\phi \uparrow D)$ if $x \in D^{\circ}$ for some $D \in D_{0}$, and $(\operatorname{det} \phi)(x)=0$ otherwise.

6.1. Proposition. Let $A$ be an interval, and let $\phi: A \rightarrow \mathbf{R}^{m}$ be an admissible injection such that $\phi(A)$ is again an interval. If $f$ is an integrable function on $\phi(A)$, then $f \circ \phi \cdot|\operatorname{det} \phi|$ is integrable on $A$ and $\int_{A} f \circ \phi \cdot|\operatorname{det} \phi|=\int_{\phi(A)} f$.

ProOF. In view of 3.4 and 3.6, we lose no generality by assuming that $\phi$ is an affine map of $\mathbf{R}^{m}$. Since $B=\phi(A)$ is an interval, there exist $a=\left(\alpha_{1}, \ldots, \alpha_{m}\right)$ and $b=\left(\beta_{1}, \ldots, \beta_{m}\right)$ in $\mathbf{R}^{m}$ such that $\prod_{i=1}^{m} \alpha_{i} \neq 0$, and $\phi(x)=a \cdot x+b$ for each $x \in \mathbf{R}^{m}$. In particular, $\phi$ maps intervals onto intervals, and $k$-planes onto $k$-planes. Let $\alpha=$ $\prod_{i=1}^{m} \alpha_{i}$ and $\rho=|\alpha| /|a|^{m}$. By 4.2, we may assume that $(\operatorname{det} \phi)(x)=\alpha$ for each $x \in$ $A$. Choose a regulator $(\varepsilon, \not H)$, and let $\mathscr{H}^{*}=\{\phi(H): H \in \not{H}\}$. Then $\left(\rho \varepsilon, \mathscr{H}^{*}\right)$ is also a regulator, and we can find a $\delta^{*}: B \rightarrow \mathbf{R}_{+}$so that $\left|\sigma(f, Q)-\int_{B} f\right|<\rho \varepsilon$ for each $Q \in$ $\mathcal{P}\left(B ; \rho \varepsilon, H^{*} ; \delta^{*}\right)$. Set $\delta=\left(\delta^{*} \circ \phi\right) /|a|$ and select a $P=\left\{\left(A_{1}, x_{1}\right), \ldots,\left(A_{p}, x_{p}\right)\right\}$ in $\mathcal{P}(A ; \varepsilon, \not{H} ; \delta)$. Now it is easy to check that $Q=\left\{\left[\phi\left(A_{1}\right), \phi\left(x_{1}\right)\right], \ldots,\left[\phi\left(A_{p}\right), \phi\left(x_{p}\right)\right]\right\}$ is in $P\left(B ; \rho \varepsilon, H^{*} ; \delta^{*}\right)$, and hence

$$
\begin{aligned}
\left|\sigma(f \circ \phi \cdot|\operatorname{det} \phi|, P)-\int_{B} f\right|=\left|\sum_{i=1}^{p} f \circ \phi\left(x_{i}\right)\right| \alpha|| A_{i}\left|-\int_{B} f\right| \\
\quad=\left|\sum_{i=1}^{p} f\left[\phi\left(x_{i}\right)\right]\right| \phi\left(A_{i}\right)\left|-\int_{B} f\right|=\left|\sigma(f, Q)-\int_{B} f\right|<\rho \varepsilon \leq \varepsilon .
\end{aligned}
$$

We show next that for $m=2$, admissible injections cannot be replaced by piecewise linear injections in Proposition 6.1. 
6.2. EXAMPLE. Let $m=2, A=[-3,3] \times[-3,3]$, and for $n=1,2, \ldots$, let

$$
A_{+}^{n}=\left[3 \cdot 2^{-n-1}, 2^{-n+1}\right] \times\left[2^{-n-1}, 2^{-n}\right]
$$

and

$$
A_{-}^{n}=\left[2^{-n-1}, 2^{-n}\right] \times\left[3 \cdot 2^{-n-1}, 2^{-n+1}\right] .
$$

Given $x \in A$, set $f(x)= \pm 2^{2(n+1)} / n$ if $x \in A_{ \pm}^{n}$, and $f(x)=0$ otherwise. Finally, let $x_{0}=(0,0)$.

We claim that $f \in R(A)$ and $\int_{A} f=0$. To see this, choose $\delta_{k}: A \rightarrow \mathbf{R}_{+}$, $k=1,2, \ldots$, so that $\delta_{k}\left(x_{0}\right)<2^{-k}$, and if $x \in A-\left\{x_{0}\right\}$, then $\delta_{k}(x)<|x|$ and $\delta_{k}(x)<\operatorname{dist}\left(x, A_{ \pm}^{n}\right)$ for each $A_{ \pm}^{n}$ which does not contain $x$. Now if $P$ is a $\delta_{k}$-fine partition of $A$, then it is not hard to show that

$$
|\sigma(f, P)|=\sum\left\{\int_{D} f:(D, x) \in P, x \neq x_{0}\right\}<\frac{k}{k+1} .
$$

There is a piecewise linear bijection $\phi: A \rightarrow A$ such that $\phi$ restricted to $C=$ $[0, \sqrt{2}] \times[0, \sqrt{2}]$ is the rotation by $-\pi / 4$ about $x_{0}$. Defining $\operatorname{det} \phi$ in the obvious way, we claim that $f \circ \phi \cdot|\operatorname{det} \phi|$ is not integrable on $A$. Indeed, if it were, then by 3.4 and $4.2, f \circ \phi$ would be integrable on $C$. However this contradicts 4.6; for $f \circ \phi \geq 0$ on $C$ and (L) $\int_{C} f \circ \phi=\sum_{n=1}^{\infty}(1 / n)=+\infty$.

6.3. REMARK. It is clear that examples similar to the previous one can be constructed for any $m \geq 1$. It follows that except for $m=1$, the integral we have defined is tied to the affine structure of $\mathbf{R}^{m}$. Fortunately, it appears that by using convex linear cells instead of intervals, we may be able to modify Definition 3.1 so that the resulting integral is invariant with respect to piecewise linear homeomorphisms, and still integrates the divergence of every differentiable vector field. As the regularity relative to a plane is meaningless for convex linear cells (e.g., it may be arbitrarily small for a simplex whose absolute regularity is $1 / m)$, we have to proceed along the lines suggested in $\left[\mathbf{P}_{\mathbf{1}}\right]$. The details will appear elsewhere.

7. Discussion. In the previous sections, we showed that our definition of the integral yields satisfactory results. However, there is no obvious reason why a different, possibly simpler, definition would not produce as good results as those we have obtained. Thus it seems much in place to provide some motivation for Definition 3.1, which is too complicated to be self-motivating. We shall do this indirectly, by presenting various modifications of Definition 3.1 and pointing out (often without proofs) their consequences. As obtaining the divergence theorem has been the principal task of this paper, we shall consider only those modifications for which Theorem 5.4 remains valid.

We begin with a modification leading to the GP-integral of Mawhin (see $\left[\mathbf{M}_{\mathbf{2}}\right.$, Definition 9]), and show by example that Proposition 3.6 does not hold for this integral.

7.1. If $f$ is a function on an interval $A$, then $I(f, A)$ denotes a unique number with the following property: given $\varepsilon>0$, there is a $\delta: A \rightarrow \mathbf{R}_{+}$such that $\mid \sigma(f, P)-$ $I(f, A) \mid<\varepsilon$ for each $P \in \mathcal{P}(A ; \varepsilon, \varnothing ; \delta)$. 
7.2. EXAMPle. Let $m=2, A=[0,1] \times[0,1], B=[-1,0] \times[0,1]$, and for $n=1,2, \ldots$, let $A_{+}^{n}=\left[3 \cdot 2^{-n-1}, 2^{-n+1}\right] \times\left[0,2^{-2 n}\right]$ and $A_{-}^{n}=\left[0,2^{-2 n}\right] \times$ $\left[3 \cdot 2^{-n-1}, 2^{-n+1}\right]$. Given $x \in A \cup B$, set $f(x)= \pm 2^{3 n+1} / n$ if $x \in A_{ \pm}^{n}$, and $f(x)=0$ otherwise. Finally, let $x_{0}=(0,0)$.

Clearly $I(f, B)=0$, and we claim that also $I(f, A)=0$. To see this, choose $\varepsilon>0$ and an integer $k \geq 1$ with $k /\left(k^{2}+1\right)<\varepsilon$. Next find $\delta: A \rightarrow \mathbf{R}_{+}$so that $\delta\left(x_{0}\right)<2^{-k^{2}}$, and if $x \in A-\left\{x_{0}\right\}$, then $\delta(x)<|x|$ and $\delta(x)<\operatorname{dist}\left(x, A_{ \pm}^{n}\right)$ for each $A_{ \pm}^{n}$ which does not contain $x$. Now if $P \in P(A ; \varepsilon, \varnothing ; \delta)$, then it is not difficult to check that

$$
\begin{aligned}
|\sigma(f, P)| & =\left|\sum\left\{\int_{C} f:(C, x) \in P, x \neq x_{0}\right\}\right| \\
& \leq \sum_{n=k^{2}+1}^{k^{2}+k} \frac{1}{n} \leq \frac{k}{k^{2}+1}<\varepsilon .
\end{aligned}
$$

On the other hand, given $\delta: A \cup B \rightarrow \mathbf{R}_{+}$, find an integer $k \geq 1$ so that $2^{-k+1}<\delta\left(x_{0}\right)$, and let $C_{0}=\left[0,2^{-k+1}\right] \times\left[0,2^{-k+1}\right]$ and $D_{0}=\left[2^{-2 k}-2^{-k+1}, 2^{-2 k}\right] \times$ $\left[0,2^{-k+1}\right]$. Now it is easy to find partitions $P=\left\{\left(C_{0}, x_{0}\right),\left(C_{1}, y_{1}\right), \ldots,\left(C_{p}, y_{p}\right)\right\}$ and $Q=\left\{\left(D_{0}, x_{0}\right),\left(D_{1}, z_{1}\right), \ldots,\left(D_{q}, z_{q}\right)\right\}$ in $P(A \cup B ; 1, \varnothing ; \delta)$ such that $\sigma(f, P)=$ $\sum_{i=1}^{p} \int_{C_{i}} f=0$ and

$$
\sigma(f, Q)=\sum_{j=1}^{q} \int_{D_{j}} f=\sum_{n=k}^{2 k-1} \frac{1}{n} \geq \frac{k}{2 k-1}>\frac{1}{2} .
$$

It follows that $I(f, A \cup B)$ does not exist.

7.3. REMARK. If in 7.1 we replace $P(A ; \varepsilon, \varnothing ; \delta)$ by $P(A ; r(A), \varnothing ; \delta)$, we obtain a modification which is similar to the $R P$-integral of Mawhin (see $\left[\mathbf{M}_{2}\right.$, Definition 10] or $\left.\left[\mathbf{M}_{\mathbf{1}}\right]\right)$. For this integral neither Proposition 3.6 nor Proposition 3.4 hold. This can be seen as follows. In the notation of Example 7.2, given $x \in A$, set $g(x)= \pm n 2^{3 m+1}$ if $x \in A_{ \pm}^{n}$, and $g(x)=0$ otherwise. Then observe that $g$ is integrable on $A$ but not on $A \cup B$ or $\left[0, \frac{1}{2}\right] \times[0,1]$. It is immediate that the same happens when the actual RP-integral is used.

7.4. REMARK. Example 7.2 indicates that $(\varepsilon, \mathscr{H})$-partitions are unavoidable. However, there is a natural alternative which would allow replacing $(\varepsilon, \mathcal{K})$-partitions by $(\varepsilon, \varnothing)$-partitions and yet keep Proposition 3.6 valid. Namely, call a partition $\left\{\left(A_{1}, x_{1}\right), \ldots,\left(A_{p}, x_{p}\right)\right\}$ special if $x_{i}$ is a vertex of $A_{i}, i=1, \ldots, p$, and then use only special partitions in Definition 3.1. Unfortunately, given an interval $A$, the author has not been able to establish whether there exists an $\varepsilon>0$ such that $\mathrm{P}(A ; \varepsilon, \varnothing ; \delta)$ contains a special partition for each $\delta: A \rightarrow \mathbf{R}_{+}$.

NOTE. Say that a function $f$ on an interval $A$ is $I^{*}$-integrable whenever there exists a division $D$ of $A$ such that $f$ is $I$-integrable on each $D \in D$, and set $I^{*}(f, A)=$ $\sum_{D \in D} I(f, D)$. It is easy to verify that the value of $I^{*}(f, A)$ does not depend on the choice of $D$, and that Proposition 3.6 is valid for the integral $I^{*}$. A serious shortcoming of this simplistic restoration of Proposition 3.6 is the complete failure of Proposition 4.10. Indeed, in Example 7.2, the squares

$$
B_{n}=\left[2^{-2 n}-2^{-n+1}, 2^{-2 n}\right] \times\left[0,2^{-n+1}\right],
$$


$n=1,2, \ldots$, converge to $x_{0}$, and yet

$$
\begin{aligned}
\lim I^{*}\left(f, B_{n}\right) & =\lim I\left(f,\left[0,2^{-2 n}\right] \times\left[0,2^{-n+1}\right]\right) \\
& =-\sum_{k=n}^{2 n} \frac{1}{k} \leq-\frac{n+1}{2 n}<-\frac{1}{2} .
\end{aligned}
$$

As it stands, Proposition 4.10 fails also for the integral $I$, however, the following weaker version of it remains correct. If $f$ is an $I$-integrable function on an interval $A$ and a sequence $\left\{B_{n}\right\}$ of subintervals of $A$ converges to an $\{x\} \subset A$, then $\lim I\left(f, B_{n}\right)=0$ whenever $\inf r\left(B_{n}\right)>0$.

7.5. If $f$ is a function on an interval $A$, then $\int_{A}^{*} f$ denotes a unique number with the following property: given a regulator $(\varepsilon, \mathcal{H})$ and $\alpha: A \rightarrow \mathbf{R}_{+}$, there is a $\delta: A \rightarrow \mathbf{R}_{+}$such that $\left|\sigma(f, P)-\int_{A}^{*} f\right|<\varepsilon$ for each $P \in P(A ; \alpha, \nvdash ; \delta)$.

The next lemma shows that the modification of 7.5 coincides with Definition 3.1, i.e., $\int^{*}=\int$.

7.6. LEMMA. Let $f$ be an integrable function on an interval $A$, and let $(\varepsilon, \not H)$ be a regulator. Then given $\alpha: A \rightarrow \mathbf{R}_{+}$, there is a $\delta: A \rightarrow \mathbf{R}_{+}$such that $\mid \sigma(f, P)-$ $\int_{A} f \mid<\varepsilon$ for each $P \in \mathcal{P}(A ; \alpha, \mathcal{H} ; \delta)$.

ProOF. By 4.3 , there are $\delta_{n}: A \rightarrow \mathbf{R}_{+}$such that

$$
\sum_{i=1}^{q}\left|f\left(x_{i}\right)\right| A_{i}\left|-\int_{A_{i}} f\right|<\varepsilon 2^{-n}
$$

for each $\left\{\left(A_{1}, x_{1}\right), \ldots,\left(A_{q}, x_{q}\right)\right\}$ in $\mathcal{P}\left(A ; 1 / n, \not H ; \delta_{n}\right), n=1,2, \ldots$. The interval $A$ is a disjoint union of the sets $E_{1}=\{x \in A: \alpha(x) \geq 1\}$ and $E_{n}=\{x \in A: 1 / n \leq$ $\alpha(x)<1 /(n-1)\}, n=2,3, \ldots$ For $x \in A$ set $\delta(x)=\delta_{n}(x)$ if $x \in E_{n}$, and choose a $P=\left\{\left(B_{1}, y_{1}\right), \ldots,\left(B_{p}, y_{p}\right)\right\}$ in $\mathcal{P}(A ; \alpha, \mathscr{H} ; \delta)$. By 2.5 , each $P_{n}=\left\{\left(B_{i}, y_{i}\right): y_{i} \in E_{n}\right\}$ is a subset of some $Q \in P\left(A ; 1 / n, \mathscr{H} ; \delta_{n}\right)$, and clearly $P=\bigcup_{n=1}^{k} P_{n}$ for some integer $k \geq 1$. Consequently,

$$
\begin{aligned}
\left|\sigma(f, P)-\int_{A} f\right| & =\sum_{n=1}^{k} \sum_{y_{i} \in E_{n}}\left|f\left(y_{i}\right)\right| B_{i}\left|-\int_{B_{i}} f\right| \\
& <\sum_{n=1}^{k} 2^{-n} \varepsilon<\varepsilon .
\end{aligned}
$$

7.7. Fix an $\alpha: \mathbf{R}^{m} \rightarrow(0,1)$. If $f$ is a function on an interval $A$, then $I_{\alpha}(f, A)$ and $I_{*}(f, A)$ denote unique numbers with the following properties: given a regulator $(\varepsilon, \not{H})$, there is a $\delta: A \rightarrow \mathbf{R}_{+}$such that $\left|\sigma(f, P)-I_{\alpha}(f, A)\right|<\varepsilon$ for each $P \in$ $P(A ; \alpha, \sharp ; \delta)$ and $\left|\sigma(f, Q)-I_{*}(f, A)\right|<\varepsilon$ for each $Q \in P(A ; 1-\delta, \nLeftarrow ; \delta)$.

The integrals $I_{\alpha}$ and $I_{*}$ are well behaved, although it is not clear whether they satisfy Proposition 6.1. Obviously, $I_{*}(f, A)$ exists whenever $I_{\alpha}(f, A)$ exists, and $I_{\alpha}(f, A)$ exists for each $f \in R(A)$. However, it is not known which of these implications, if either, can be reversed.

To see some more sophisticated modifications, we cast Definition 3.1 in the form of a variational integral (see $\left[\mathbf{H}_{\mathbf{1}}\right]$ ).

Let $A$ be an interval. A superadditive function in $A$ is a function $F$ of subintervals of $A$ such that $F(B) \geq \sum_{D \in D} F(D)$ for each interval $B \subset A$ and each division $D$ 
of $B$. Replacing the sign $\geq$ by $=$, we obtain the definition of an additive function in $A$. If $f \in R(A)$, then by 3.4 and 3.6, the function $B \mapsto \int_{B} f$ of subintervals of $A$ is additive; we denote it by $\int f$.

7.8. DEFINITION. Let $\not$ be a family of planes, let $f$ and $\varepsilon$ be functions on an interval $A$, and let $F$ and $M$ be functions of subintervals of $A$. We say that $M$ is an $(\varepsilon, \mathcal{H})$-majorant of the pair $(f, F)$ if there is a $\delta: A \rightarrow \mathbf{R}_{+}$such that $|f(x)| B|-F(B)| \leq M(B)$ for each interval $B \subset A$ with $x \in B, d(B)<\delta(x)$, and $r(B ; \not{A}) \geq \varepsilon(x)$.

Note that if $M$ is a superadditive $(\varepsilon, \not H)$-majorant and $\varepsilon<1$, then $0 \leq M \leq$ $M(A)$. Indeed, by Corollary 2.5, each interval $C \subset A$ has a $\delta$-fine $(\varepsilon, \not /)$-partition $\left\{\left(C_{1}, x_{1}\right), \ldots,\left(C_{p}, x_{p}\right)\right\}$, and hence

$$
M(C) \geq \sum_{i=1}^{p} M\left(C_{i}\right) \geq \sum_{i=1}^{p}\left|f\left(x_{i}\right)\right| C_{i}\left|-F\left(C_{i}\right)\right| \geq 0 .
$$

From this and the superadditivity of $M$, we see that $M \leq M(A)$.

7.9. Proposition. Let $f$ be a function on an interval $A$. Then $f \in R(A)$ if and only if there is an additive function $F$ in $A$ such that for each regulator $(\varepsilon, \mathcal{H})$ the pair $(f, F)$ has a superadditive $(\varepsilon, \not H)$-majorant $M$ with $M(A)<\varepsilon$; in which case $F=\int f$.

ProOF. Suppose that an additive function $F$ in $A$ satisfying the condition of the proposition exists. Given a regulator $(\varepsilon, \not{H})$, find a superadditive $(\varepsilon, \mathscr{H})$-majorant $M$ of $(f, F)$ with $M(A)<\varepsilon$, and choose the corresponding $\delta: A \rightarrow \mathbf{R}_{+}$. Now let $B$ be a subinterval of $A$, and let $P=\left\{\left(B_{1}, x_{1}\right), \ldots,\left(B_{p}, x_{p}\right)\right\}$ be in $P(B ; \varepsilon, \mathscr{H} ; \delta)$. In view of the note following Definition 7.8 , we have

$$
\begin{aligned}
|\sigma(f, P)-F(B)| & \leq \sum_{i=1}^{p}\left|f\left(x_{i}\right)\right| B_{i}\left|-F\left(B_{i}\right)\right| \\
& \leq \sum_{i=1}^{p} M\left(B_{i}\right) \leq M(B)<\varepsilon .
\end{aligned}
$$

It follows that $f \in R(B)$ and $\int_{B} f=F(B)$.

Conversely, suppose that $f \in R(A)$ and let $F=\int f$. Given a regulator $(\varepsilon, \not{K})$, use 4.3 to find a $\delta: A \rightarrow \mathbf{R}_{+}$so that $|\sigma(f, P)-F(B)|<\varepsilon / 3$ for each interval $B \subset A$ and each $P \in P(B ; \varepsilon, H ; \delta)$. This is possible, for by $2.5, P$ is a subset of some $Q \in \mathcal{P}(A ; \varepsilon, \not H ; \delta)$. Now for every interval $B \subset A$, set

$$
G(B)=\inf \sigma(f, P) \text { and } H(B)=\sup \sigma(f, P)
$$

where the infimum and supremum are taken ever all $P \in P(B ; \varepsilon, \not \nvdash ; \delta)$. By 2.5 and our choice of $\delta$, we have $F-\varepsilon / 3 \leq G \leq H \leq F+\varepsilon / 3$. Thus if $M=H-G$, then $0 \leq M \leq 2 \varepsilon / 3<\varepsilon$. Let $B$ be a subinterval of $A$, and let $\alpha>0$. By 3.4, there is a $P \in P(B ; \varepsilon, \nvdash ; \delta)$ such that $F(B)-\alpha<\sigma(f, P)<F(B)+\alpha$. Consequently, $F(B)-\alpha<H(B)$ and $G(B)<F(B)+\alpha$, and by the arbitrariness of $\alpha$, also $G(B) \leq F(B) \leq H(B)$. Next assume that $x \in B, d(B)<\delta(x)$, and $r(B ; \not H) \geq \varepsilon$. then $\{(B, x)\}$ belongs to $P(B ; \varepsilon, \not H ; \delta)$, and so $G(B) \leq f(x)|B| \leq$ $H(B)$. Therefore $|f(x)| B|-F(B)| \leq M(B)$, and we see that $M$ is an $(\varepsilon, \mathcal{H})$ majorant of $(f, F)$. It remains to show that $M$ is superadditive. To this end, let 
$\left\{C_{1}, \ldots, C_{k}\right\}$ be a division of an interval $C \subset A$, and let $P_{i} \in \mathcal{P}\left(C_{i} ; \varepsilon, \mathcal{H} ; \delta\right), i=$ $1, \ldots, k$. Then $P=\bigcup_{i=1}^{k} P_{i}$ belongs to $P(C ; \varepsilon, \not H ; \delta)$ and $\sigma(f, P)=\sum_{i=1}^{k} \sigma\left(f, P_{i}\right)$. Hence $G(C) \leq \sum_{i=1}^{k} \sigma\left(f, P_{i}\right) \leq H(C)$, and by the arbitrariness of the $P_{i}$ 's, also $G(C) \leq \sum_{i=1}^{k} G\left(C_{i}\right)$ and $H(C) \geq \sum_{i=1}^{k} H\left(C_{i}\right)$. The superadditivity of $M$ follows.

Observe that, in the respective notations of the proofs of Proposition 4.1 and Theorem 5.4, the following holds:

(i) If $F(B)=(\mathrm{L}) \int_{B} f$ and

$$
M(B)=\frac{\varepsilon|B|}{2|A|}+(\mathrm{L}) \int_{B}(h-g)
$$

for each interval $B \subset A$, then $M(A)<\varepsilon$ and $M$ is an additive $(0, \varnothing)$-majorant of $(f, F)$.

(ii) If $G(B)=\left(\mathrm{L}_{m-1}\right) \int_{\partial B} v \cdot n$ and

$$
N(B)=\varepsilon\left(|B|+\sum_{k=0}^{m-1} \sum_{n=1}^{\infty} 2^{-n}\left|B \cap H_{k}^{n}\right|_{k}\right)
$$

for each interval $B \subset A$, then $N(A)<\varepsilon d^{m}(1+m)$ and $N$ is an additive $(\alpha, \nvdash)$ majorant of $(\nabla \cdot v, G)$ for each $\alpha>0$.

Proposition 7.9 together with the above observations suggest the following modifications of Definition 3.1.

7.10. If $f$ is a function on an interval $A$, then $J(f)$ denotes a unique additive function in $A$ such that for each regulator $(\varepsilon, \not)$ the pair $[f, J(f)]$ has an additive $(\varepsilon, \mathcal{H})$-majorant $M$ with $M(A)<\varepsilon$.

7.11. If $f$ is a function on an interval $A$, then $J_{s}(f)$ and $J_{a}(f)$ are unique additive functions in $A$ with the following properties: given a regulator $(\varepsilon, \not H)$, in $A$ there exist a superadditive function $M_{s}$ with $M_{s}(A)<\varepsilon$ and an additive function $M_{a}$ with $M_{a}(A)<\varepsilon$ such that for each $\alpha>0, M_{s}$ is an $(\alpha, \not)$-majorant of $\left[f, J_{s}(F)\right]$ and $M_{a}$ is an $(\alpha, \mathscr{H})$-majorant of $\left[f, J_{a}(f)\right]$.

The integrals $J, J_{s}$, and $J_{a}$ are well behaved; however, it is unclear whether their definitions can be translated back to those of a Riemann type. An argument similar to the proof of Lemma 7.6 shows that no new modifications are obtained from 7.10 and 7.11 by considering $(\beta, \not H)$-majorants with nonconstant $\beta$.

For an interval $A$, denote by $J(A), J_{s}(A)$, and $J_{a}(A)$ the families of all $J_{-}, J_{s^{-}}$, and $J_{a}$-integrable functions on $A$, respectively. Then $J_{a}(A) \subset J_{s}(A) \cap J(A)$ and $J_{s}(A) \cup J(A) \subset R(A)$, and the corresponding integrals coincide on the intersections of their respective domains. Whether any of the families $J(A), J_{s}(A), J_{a}(A)$, or $R(A)$ are equal to each other is unknown.

If $B$ and $C$ are intervals in $\mathbf{R}^{k}$ and $\mathbf{R}^{l}$, respectively, then following the proof of $\left[\mathbf{H}_{\mathbf{4}}\right.$, Theorem 1], we can show that $J(B) \otimes J(C) \subset J(B \times C)$ and $J(f \otimes g)=$ $J(f) \cdot J(g)$ for each $f \in J(B)$ and $g \in J(C)$. While the same holds for $J_{a}$, it is not clear whether this is also true for $J_{s}$ or for our main integral defined in 3.1. The differences between integrals defined by means of additive and superadditive majorants are discussed in $\left[\mathbf{H}_{\mathbf{2}}\right]$.

ADDED IN PROOF. The deficiency in the additive properties of Mawhin's integrals (see $\left[\mathbf{M}_{\mathbf{1}}\right.$ and $\mathbf{M}_{\mathbf{2}}$ ]) has been corrected in [JKS]. However, the divergence theorem (Theorem 3) proved in [JKS] is still substantially weaker than our Theorem 5.4. 


\section{REFERENCES}

[H1] R. Henstock, Theory of integration, Butterworths, London, 1963.

$\left[\mathrm{H}_{2}\right]$ _ Majorants in variational integration, Canad. J. Math. 18 (1966), 49-74.

$\left[\mathrm{H}_{3}\right] \longrightarrow$, A Riemann-type integral of Lebesgue power, Canad. J. Math. 20 (1968), 79-87.

$\left[\mathbf{H}_{4}\right] \ldots$, A problem in two dimensional integration, J. Austral. Math. Soc. (Ser. A) 35 (1983), $386-404$.

[JK] J. Jarník and J. Kurzweil, A nonabsolutely convergent integral which admits transformation and can be used for integration on manifolds, Czechoslovak Math. J. 35 (1985), 116-139.

[JKS] J. Jarník, J. Kurzweil and S. Schwabik, On Mawhin's approach to multiple nonabsolutely convergent integral, Casopis Pest. Mat. 108 (1983), 356-380.

[K1] J. Kurzweil, Generalized ordinary differential equations and continuous dependence on a parameter, Czechoslovak Math. J. 7 (1957), 418-446.

$\left[\mathbf{K}_{\mathbf{2}}\right]-$, Nichtabsolut Konvergente Integrale, Teubner, Leipzig, 1980.

[KN] L. Kuipers and H. Niederreiter, Uniform distribution of sequences, Wiley, New York, 1974.

[LW] Lee Peng Yee and Wittaya Naak-In, A direct proof that Henstock and Denjoy integrals are equivalent, Bull. Malaysian Math. Soc. (2) 5 (1982), 43-47.

[M1] J. Mawhin, Generalized Riemann integrals and the divergence theorem for differentiable vector fields, in E. B. Christoffel, Birkhäuser-Verlag, Basel, 1981, pp. 704-714.

$\left[\mathbf{M}_{2}\right]$, Generalized multiple Perron integrals and the Green-Goursat theorem for differentiable vector fields, Czechoslovak Math. J. 31 (1981), 614-632.

[Mc] R. M. McLeod, The generalized Riemann integral, Carus Math. Monographs, 20, Math. Assoc. Amer., 1980.

[Mu] J. R. Munkres, Elementary differential topology, Princeton Univ. Press, Princeton, N.J., 1966.

[P1] W. F. Pfeffer, Une intégrale riemannienne et le théorème de divergence, Analyse Math., C. R. Acad. Sci. Paris Sér. I 299 (1984).

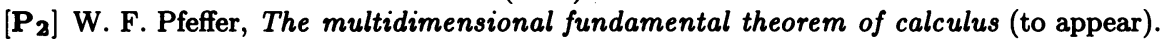

[R] W. Rudin, Principles of mathematical analysis, McGraw-Hill, New York, 1976.

[S] S. Saks, Theory of the integral, Dover, New York, 1964.

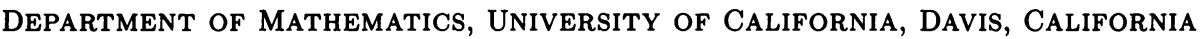
95616 